\title{
Rôle du facteur SF-1 dans le développement des gonades et des surrénales, et dans la stéroïdogenèse
}

\begin{abstract}
Dans le cortex surrénalien et dans les gonades, la production de stéroïdes à partir du cholestérol implique plusieurs étapes catalysées par des enzymes spécifiques dont la structure des gènes et la localisation chromosomique ont été élucidées [1,2]. Les hormones spécifiques, corticotropine $(\mathrm{ACTH})$ pour la surrénale [3] et gonadotrophines pour les gonades [4], gouvernent, par l'intermédiaire de l'AMPc, l'expression des gènes codant pour ces enzymes. Bien que le promoteur de la plupart de ces gènes ne contienne pas de séquence consensus CRE (cAMP regulatory element), des séquences contrôlées par l'AMPc (CRS, cAMP responsive sequences) ont été identifiées. La localisation et la séquence de ces éléments sont non seulement spécifiques de chaque gène, mais aussi, pour le même gène, spécifiques de tissu et d'espèce.
\end{abstract}

Plus récemment, il a été montré que tous les cytochromes P450 codant pour les enzymes de la stéroïdogenèse (P450scc, P450 17 $\alpha$-hydroxylase, P450 aromatase, P450 21-hydroxylase, P450 11 $\beta$-hydroxylase et P450 aldostérone synthase) contiennent une ou plusieurs séquences consensus $(\mathrm{C} / \mathrm{T})$ CAA GG (T/C). Cette séquence lie un facteur nucléaire appelé SF-1 (ste- roidogenic factor 1) [5] ou $\mathrm{Ad} 4 \mathrm{BP}$ (adrenal 4-binding protein) [6]. La protéine a été purifiée à partir de surrénales bovines et les ADNc murin et bovin codant pour cette protéine clonés [6-9]. L'utilisation des anticorps spécifiques [10] et l'étude de l'ARNm par Northern blot, RT-PCR et hybridation in situ [8, 10-12], ont montré que le gène de ce facteur s'exprime dans tous les tissus stéroïdogènes (cortex surrénal, cellules de Leydig, cellules thécales, cellules de la granulosa et lutéales), dans les cellules de Sertoli et, à un moindre degré, dans le placenta, les adipocytes, l'hypophyse et le cerveau. Au cours de la vie fotale, son expression dans la crête urogénitale est très précoce et apparaît vers le $9^{\mathrm{e}}$ jour de gestation [12].

L'analyse de l'ADNc du SF-1 a révélé que celui-ci code pour une protéine qui a une grande similitude avec les membres de la superfamille des récepteurs des hormones stéroïdes, avec un doigt de zinc dans la partie $\mathrm{N}$-terminale et un domaine de liaison/dimérisation dans la partie C-terminale. Par ailleurs, certains domaines du gène $S F-1$ sont identiques à ceux de l'ADNc ELP (embryonal long terminal receptor-binding protein) isolé d'une banque d'ADNc préparée à partir de cellules de carcinome embryonnaire de souris [13]. L'isolement et la caractérisation des clones génomiques ont montré que SF-1 et ELP dérivent du même gène, mais avec l'utilisation d'un promoteur et d'un épissage différents [11]. La deuxième homologie qui a été trouvée est avec le gène FTZ-F1 (fushi tarazu factor 1) de la drosophile qui code pour un récepteur nucléaire orphelin gouvernant l'expression de l'homéoboîte fushi tarazu pendant le développement précoce $[14,15]$. Comme chez la souris, le gène de la drosophile code pour deux transcrits différents [16].

Outre le rôle important du SF-1 dans l'expression des cytochromes P450 codant pour les enzymes de la stéroïdogenèse, deux travaux récents montrent que ce facteur joue un rôle crucial dans le développement embryonnaire du cortex surrénal et des gonades $(\mathrm{m} / \mathrm{s}$ $n^{\circ} 10$, vol. 10, p. 1055), et probablement des cellules gonadotropes hypophysaires. Chez la souris, l'invalidation du gène $S F-1$ par recombinaison homologue produit, chez les homozygotes, une agénésie complète des surrénales et des gonades, avec des organes génitaux externes et internes de type féminin [17]. La présence, chez les souris mâles, des or- 
ganes génitaux internes de type féminin suggère l'absence d'hormone anti-müllerienne (AMH) responsable, chez le mâle, de la régression des canaux de Müller [18, 19]. Dans le deuxième travail [20], il est montré que SF-1, qui est exprimé dans les cellules de Sertoli fœtales [12], règle l'expression de l'AMH dans ces cellules. L'expression du SF-l précède celle de l'AMH et, après la différenciation des gonades, l'expression du SF-1, comme celle d'AMH, est plus importante chez le fœtus mâle que chez le fœtus femelle. Ces résultats montrent donc que SF-1, en plus d'être un activateur de l'expression des gènes spécifiques des cellules stéroïdogènes et de Sertoli, est nécessaire à la différenciation de ces cellules. Il peut être comparé à un autre facteur transcriptionnel du système endocrine, Pit-1, nécessaire au développement des cellules somatotropes de l'hypophyse et à l'expression de l'hormone de croissance et de la prolactine [21].

Un travail récent [22] a montré que le facteur GSEB-1 (gonadotropin-specific element binding protein 1), présent dans les cellules gonadotropes et dans une lignée cellulaire dérivée de ces cellules, est en fait SF-1. Dans le même travail, a été montrée l'augmentation de la transcription de constructions contenant un ou deux GSE et un gène rapporteur dans des cellules (gonadotropes ou stéroïdogènes) exprimant SF-1, suggérant que ce facteur est actif en trans et règle l'expression de la sous-unité $\alpha$ des gonadotrophines dans les cellules gonadotropes. Étant donné que la sous-unité $\alpha$ est un des premiers gènes hormonaux exprimé dans l'hypophyse (avant le $13^{\mathrm{e}}$ jour de vie foetale chez la souris), SF-1 pourrait être, non seulement un facteur activant la transcription de ce gène, mais aussi un facteur contrôlant la différenciation des cellules gonadotropes. L'absence de cellules gonadotropes dans l'hypophyse des animaux dont le gène $S F$ - 1 a été invalidé [23] pourrait être en faveur de cette hypothèse. Cependant, le problème semble plus complexe : en effet, par hybridation in situ, les auteurs ont montré l'absence de récepteur de la LHRH

dans l'hypophyse, et pourtant le traitement des animaux homozygotes avec un analogue de LHRH induit l'apparition des cellules gonadotropes. Ces résultats auraient pu indiquer une absence de neurones à LHRH, mais des études immunohistochimiques ont montré la présence de ces neurones, délocalisés dans l'hypothalamus et avec une organisation atypique. Les auteurs concluent [23] que SF-1 serait nécessaire pour la connexion de neurones à LHRH avec le système porte hypophysaire. Bien que l'importance du SF-1 dans le développement et dans la différenciation du cortex surrénal et des gonades soit largement démontrée par tous ces travaux, plusieurs problèmes restent encore à élucider : (1) quels sont les facteurs qui déterminent la spécificité cellulaire de l'action du SF-1 sur l'expression de certains gènes : l'expression des cytochromes P450 21-hydroxylase et P450 11ß-hydroxylase dans la surrénale, mais pas dans les gonades et, inversement, l'expression de P450 aromatase dans les gonades mais pas dans la surrénale, ou encore l'expression de l'AMH exclusivement dans les cellules de Sertoli et les cellules ovariennes ; (2) quel est le ligand ou cofacteur endogène qui permet au SF-1 d'activer la transcription? Son activité transcriptionnelle dans les cellules non stéroïdogènes est très faible ou nulle $[9,24$, 25]. De même, dans les cellules HeLa, SF-1 est incapable d'activer l'expression d'une construction contenant le promoteur de l'AMH et le gène rapporteur luciférase, alors qu'un SF-1 ayant une délétion du domaine de liaison, est actif [20] ; (3) quels sont les facteurs qui gouvernent son expression et son activation?

\section{RÉFÉRENCES}

1. Miller W. Molecular biology of steroid hormone synthesis. Endocrinol Res 1988; 9 : 295-318.

2. Hanukoglu I. Steroidogenic enzymes : structure, function and role in regulation of steroid hormone biosynthesis. J Steroid Biochem Mol Biol 1992 ; 43 : 779-804.
3. Simpson ER, Waterman MR. Regulation of the synthesis of steroidogenic enzymes in adrenal cortical cells by ACTH. Annu Rev Physiol $1988 ; 50: 427-40$.

4. Payne AH, Youngblood GL, Sha L, Burgos-Trinidad M, Hammond SH. Hormonal regulation of steroidogenic enzyme gene expression in Leydig cells. I Steroid Biochem Mol Biol 1992 ; 43 : 895-906.

5. Rice DA, Mouw AR, Bogerd AM, Parker KL. A shared promoter element regulates the expression of three steroidogenic enzymes. Mol Endocrinol 1991 ; 5 : 1552-61.

6. Morohashi KL, Honda SL, Inamata Y, Handa $\mathrm{H}$, Omura T. A common trans-acting factor, Ad4-binding protein, to the promoters of steroidogenic P-450s. I Biol moters of steroidogenic
Chem $1992 ; 267: 17913-9$.

7. Lala DS, Rice DA, Parker KL. Steroidogenic factor 1 , a key regulator of steroidogenic enzyme expression, is the mouse homolog of fushi tarazu-factor 1. Mol Endocrinol 1992; $6: 1249-58$.

8. Honda SI, Morohashi KI, Nomura M, Takeya $\mathrm{H}$, Kitajima M, Omura T. Ad4BP regulating steroidogenic P-450 gene is a member of steroid hormone receptor subfamily. J Biol Chem 1993 ; 268 : 7494-502.

9. Lynch JP, Lala DS, Peluso JJ, Luo W, Parker KL, White BA. Steroidogenic factor 1 , an orphan nuclear receptor, regulates the expression of the rat aromatase gene in gonadal tissue. Mol Endocrinol 1993; $7: 776$ 86.

10. Morohashi $K I$, Iida $H$, Nomura $M$, Hatano O, Honda SI, Tsukiyama T, Shibata Y, Omura $T$. Functional difference between Ad4BP and ELP, and their distributions in steroidogenic tissues. Mol Endocrinol 1994 ; $8: 643-53$.

11. Ikeda Y, Lala DS, Luo X, Kim E, Moisan MP Parker KL. Characterization of the mouse, $F T Z F I$ gene, which encodes a key regulator of steroid hydroxylase gene expression. Mol Endocrinol $1993 ; 7$ : 852-60.

12. Ikeda $\mathrm{X}$, Shen WH, Ingraham HA, Parker KL. Developmental expression of mouse steroidogenic factor-1, an essential regulator of the steroid hydroxylases. Mol Endocrinol $1994 ; 8$ : 65462.

13. Tsukiyama $T$, Ueda $H$, Hirose $S$, Niwa $O$. Embryonal long terminal repeat-binding protein is a murine homolog of FTZ-F1, a member of the steroid receptor superfamily. Mol Cell Biol 1992 ; 12 : 1286-91.

14. Lavorgna G, Ueda H, Clos J, Wu C. FTZ$\mathrm{F} 1$, a steroid hormone receptor-like protein implicated in the activation of fushi tarazu. Science 1991 ; 252 : 848-51.

15. Ueda H, Sonoda S, Brown JL, Scott MP, Wu C. A sequence-specific DNA-binding protein that activates fushi tarazu segmentation gene expression. Genes Dev $1990 ; 4$ : $624-35$. 
16. Lavorgna G, Karim FD, Thummel CS, Wu C. Potential role for a FTZ-F1 steroid receptor superfamily member in the control of Drosophila metamorphosis. Proc Natl Acad Sci USA 1993 ; 90 : 3004-8.

17. Luo X, Ikeda Y, Parker KL. A cell-specific nuclear receptor is essential for adrenal and gonadal development and sexual differentiation. Cell 1994; 77 : 481-90.

18. Josso N, Picard J, Vigier B, Tran D. L.'hormone anti-müllérienne. médecine/sciences 1987 ; $3: 444-52$.

19. Josso N, Boussin L, Knebelmann B, Nihoul-Fekete C, Picard JY. Anti-Müllerian hormone and intersex states. Trends Endo cinol Metab 1991 ; 2 : 227-33.

20. Shen WH, Moore CCD, lkeda Y Parker KL, Ingraham HA. Nuclear receptor steroidogenic factor 1 regulates the Mullerian inhibiting substance gene : a link to the sex determination cascade. Cell $1994 ; 77$. $651-61$.

21. Pfaffe RW, DiMattia GE, Parks IS Brown MR, Wit JM Jansen $M$, Van der Nat $H$, Van den Brande JL, Rosenfeld MG, Ingraham HA. Mutation of the POU-specific domain of Pit-l and hypopituitarism without pituitary hypoplasia. Science 1992 ; $257: 1118-21$.

22. Barnhart KM, Mellon DL. The orphan nuclear receptor, steroidogenic factor-1, regulates the gycoprotein hormone $\alpha$-subunit gene in pituitary gonadotropes. Mol Endorrinol $1994 ; 8: 878-85$

23. Ikeda Y, I uo X, Lala DS, Shen WH, Moore CCA, Ingraham HA, Parker KL. The nuclear receptor SF-1 is essential for developpement of steroidogenic tissues. IX. International Congress on hormonal steroids. Dallas, September 2429, 1994 ; Abstract p. 68 .

24. Morohashi KI, Zanger UM, Honda SI, Hara M, Waterman MR, Omura T. Activation of CYPIIA and CYPIIB gene promoters by the steroidogenic cell-specific transcription factor Ad4BP. Mol Endocrinol 1993 $7: 1196-204$

25. Fitzpatrick SI, Richards JS. cis-acting elements of the rat aromatase promoter required for cyclic adenosine 3',5'-monophosphate induction in ovarian granulosa cells and constitutive expression in R2C L eydig cells. Mol Endocrinol $1993 ; 7: 341-54$.

\section{Jose Maria Saez}

Directeur de recherche à l'Inserm

\section{Philippe Durand}

Directeur de recherche à l'Inra

Inserm-Inra U.307, Hopital Debrousse, 29, rue Saur-Bouvier; 69322 Lyon Cedex, France.

TIRÉS À PART

J.M. Saez. 